\title{
Development of an Indirect ELISA to Detect African Swine Fever Virus pp62 Protein-Specific Antibodies
}

\author{
Kexin Zhong ${ }^{1 \dagger}$, Mengmeng Zhu ${ }^{1 \dagger}$, Qichao Yuan ${ }^{1}$, Zhibang Deng ${ }^{1}$, Simeng Feng ${ }^{1}$, \\ Daoxin Liu ${ }^{2 *}$ and Xiaomin Yuan ${ }^{1 *}$
}

${ }^{1}$ Lab of Animal Disease Prevention and Control and Animal Model, Hunan Provincial Key Laboratory of Protein Engineering in Animal Vaccines, College of Veterinary Medicine, Hunan Agricultural University (HUNAU), Changsha, China, ${ }^{2}$ Animal Disease Prevention and Control Center, Changsha, China

\section{OPEN ACCESS}

Edited by:

Valentina Virginia Ebani, University of Pisa, Italy

Reviewed by:

Semmannan Kalaiyarasu, ICAR-National Institute of High

Security Animal Diseases (ICAR-NIHSAD), India

Tereza Cristina Cardoso,

Universidade Estadual de São

Paulo, Brazil

${ }^{*}$ Correspondence: Xiaomin Yuan

yxm1230@hunau.edu.cn Daoxin Liu

719680669@qq.com

tThese authors have contributed equally to this work and share first

authorship

Specialty section This article was submitted to Veterinary Infectious Diseases,

a section of the journal

Frontiers in Veterinary Science

Received: 20 October 2021 Accepted: 13 December 2021 Published: 13 January 2022

Citation:

Zhong K, Zhu M, Yuan Q, Deng Z,

Feng S, Liu D and Yuan X (2022) Development of an Indirect ELISA to Detect African Swine Fever Virus pp62 Protein-Specific Antibodies.

Front. Vet. Sci. 8:798559 doi: 10.3389/fvets.2021.798559
African swine fever (ASF) is a highly detrimental viral disease caused by African swine fever virus (ASFV). The occurrence and prevalence of this disease have become a serious threat to the global swine industry and national economies. At present, the detection volume of African swine fever is huge, more sensitive and accurate detection techniques are needed for the market. pp62 protein, as a protein in the late stage of infection, has strong antigenicity and a high corresponding antibody titer in infected pigs. In this study, the CP530R gene was cloned into expression vector pET-28a to construct a prokaryotic expression plasmid, which was induced by IPTG to express soluble pp62 protein. Western blot analysis showed that it had great reactivity. Using the purified recombinant protein as an antigen, an indirect ELISA method for detecting ASFV antibody was established. The method was specific only to ASFV-positive serum, 1:1600 diluted positive serum could still be detected, and the coefficients of variation (CV) of the intra assay and inter assay were both $<10 \%$. It turns out that the assays had excellent specificity, sensitivity, and repeatability. This provides an accurate, rapid, and economical method for the detection of ASFV antibody in clinical pig serum samples.

\section{Keywords: African swine fever virus, indirect ELISA, CP530R gene, pp62 protein, prokaryotic expression system}

\section{INTRODUCTION}

African swine fever (ASF) is a viral disease of swine that is caused by African swine fever virus (ASFV), and mainly leads to acute, fearful, and serious contact infectious disease in domestic pigs and wild boars (1). In view of the seriousness of ASF, the World Organization for Animal Health (OIE) has listed it as a notifiable animal disease, which is also a Class 1 animal infectious disease in China (2). Furthermore, diseased pigs, rehabilitated pigs, and recessively infected pigs are the main infectious sources of the disease, and Ornithodoros soft ticks carrying ASFV are also one of the infectious sources (3).

Since ASF was first reported in Kenya, Africa, in 1921, it has been found in 60 countries across Europe, Latin America, and Asia (4). It harms the development of the swine raising industry and causes enormous economic losses in the world (5). Similarly, after the first case of ASF was reported in China in August 2018, the disease had a wide outbreak and epidemics in various regions of China, bringing tremendous economic losses to China's swine industry (2). To date, 
an effective and safe commercial ASFV vaccine is not available (6). The available methods of disease control and prevention consist of early diagnosis, rapid elimination of infectious sources, and blocking transmission routes (7). However, the number of samples to be screened in the process of early diagnosis is very large, so the required detection technology needs to have high sensitivity and accuracy. Therefore, it is of extreme significance to study the diagnostic methods of this disease. So far, there have been several routine laboratory diagnostic techniques for detecting ASFV, including polymerase chain reaction (PCR), loop-mediated isothermal amplification (LAMP), fluorescence quantitative PCR, erythrocyte adsorption (HAD), colloidal gold rapid strip, enzyme-linked immunosorbent assay (ELISA), and other serological tests. Within them, ELISA is the most commonly used method for specific antibody detection (8).

ASFV is a large, enveloped, complex icosahedral doublestranded DNA arbovirus, the sole member of the family Asfarviridae, genus Asfivirus (9). Domestic pigs, wild boars, and Ornithodoros soft ticks are the hosts of ASFV, and the virus replicates mainly in the cytoplasm of porcine monocytes and macrophages $(10,11)$. The genes of this virus have a full length of $170-190 \mathrm{~kb}$ and encode more than 200 proteins (12). Thirty-eight proteins are known to be involved in nucleotide metabolism, gene transcription, viral replication, and repair functions (13). Among them, pp62 protein is a $63.5 \mathrm{kDa}$ protein encoded by the CP530R gene. The protein sequence has high conservation and immunogenicity in different ASFV strains (14). Under the action of the S273R enzyme, the protein can be cleaved into mature viral proteins P35, P15, and P8 (15). P35 and P15 are involved in the formation of viral icosahedron and become momentous structural proteins. The mature products of pp62 are the basic components of the core and shell, and the mature products of pp220 together account for about $30 \%$ of the total protein of the virion (16). Thus, pp62 protein, as the precursor protein of structural proteins $\mathrm{P} 35$ and $\mathrm{P} 15$, may be one of the vital targets for the immunological diagnosis of African swine fever virus, and has immense significance in the basic research of the pathogenesis and immune mechanism of African swine fever virus.

In this study, we expressed and purified the ASFV pp62 protein, and established an indirect ELISA method for the detection of ASFV antibodies using pp62 protein as a diagnostic antigen, which laid the foundation for the development of ASFV early diagnosis kits and the structural and functional study of pp62 protein.

\section{MATERIALS AND METHODS}

\section{Serum Samples}

Positive standard sera and negative standard sera were obtained from the Animal Disease Prevention and Control Center (Changsha, China). The negative serum samples $(N=40)$ were decided through RT-PCR and a commercial ELISA (ID. Vet Inc., France). Clinical Serum samples $(N=350)$ were collected from sows and adult pigs on commercial swine farms from 2018 to 2021.

\section{Cloning and Expression of pp62 Protein}

The full length CP530R coding region of ASFV (GenBank accession, No.FR682468.2) was synthesized by Tsingke Biotechnology Co., Ltd. (Changsha, China) and amplified using $\mathrm{F}\left(5^{\prime}\right.$ CGGAATTCATGCCCTCTAATATGAAACAGTT) and $\mathrm{R}\left(5^{\prime}\right.$ CCAAGCTTTTATTCTTGAAGTAACTTTAGT) primers appended with EcoRI and HindIII restriction sites, respectively. A recombinant plasmid was constructed by ligating the PCR product into the vector pET-28a. The ligation product was transformed into E. coli $\mathrm{DH} 5 \alpha$ competent cells, and was verified using double restriction enzyme digestion and sequencing. E. coli BL21 competent cells were transformed with recombinant plasmid, and protein expression was induced with $1 \mathrm{mM}$ IPTG at $27^{\circ} \mathrm{C}$ for $9 \mathrm{~h}$. Nickel-nitrilotriacetic acid (Ni-NTA) metal affinity chromatography was used to purify the recombinant pp62 protein, which was identified with SDS-PAGE and Western blot. ASFV-positive pig serum was used as the primary antibody (dilution of 1:3,000) for the Western blot assay.

\section{Checkerboard Titration}

The antigen and antibody concentration were optimized using checkerboard titration. Briefly, the recombinant antigen was coated to the plate in concentrations of $0.5-2 \mu \mathrm{g} / \mathrm{mL}$. Positive and negative standard sera were diluted at 1:50-1:400. Then HRP anti-swine IgG (SeraCare, USA) was diluted at 1:5,000-1:10,000 to determine the optimal conjugate dilution. The conditions that gave the highest OD450 ratio between the positive and negative sera $(P / N$ value $)$ and an OD450 value for positive serum close to 1.0 were scored as optimal working conditions.

\section{Cut-Off Value for pp62-IELISA}

A total of 40 negative serum samples from uninfected pigs were used to calculate the cut-off value of the indirect ELISA. Statistical analysis was performed to calculate the mean value $(\bar{X})$ and standard deviation (SD) of the OD450 values. The cut-off value was determined as $\bar{X}+3 \mathrm{SD}$.

\section{Specificity and Sensitivity of pp62-IELISA}

The established pp62-iELISA assay was used against respiratory syndrome virus (PRRSV), porcine circovirus type 2(PCV2), classical swine fever virus (CSFV), pseudorabies virus (PRV), foot-and-mouth disease virus (FMDV)-positive sera as well as ASFV-positive and negative sera. ASFV-positive serum was diluted from $1: 100$ to $1: 6,400$ to determine the highest dilution of serum. The sensitivity of the ELISA was evaluated based on the cut-off.

\section{Reproducibility of pp62-IELISA}

Intra and inter-assay variation (coefficient of variation [CV]) between runs were evaluated. Briefly, five sera were randomly selected. Three replicates of each sample were assayed in one batch to evaluate intra-assay (within plate) variation and three plates were assayed as separate batches to evaluate inter-assay (between assays) variation. 


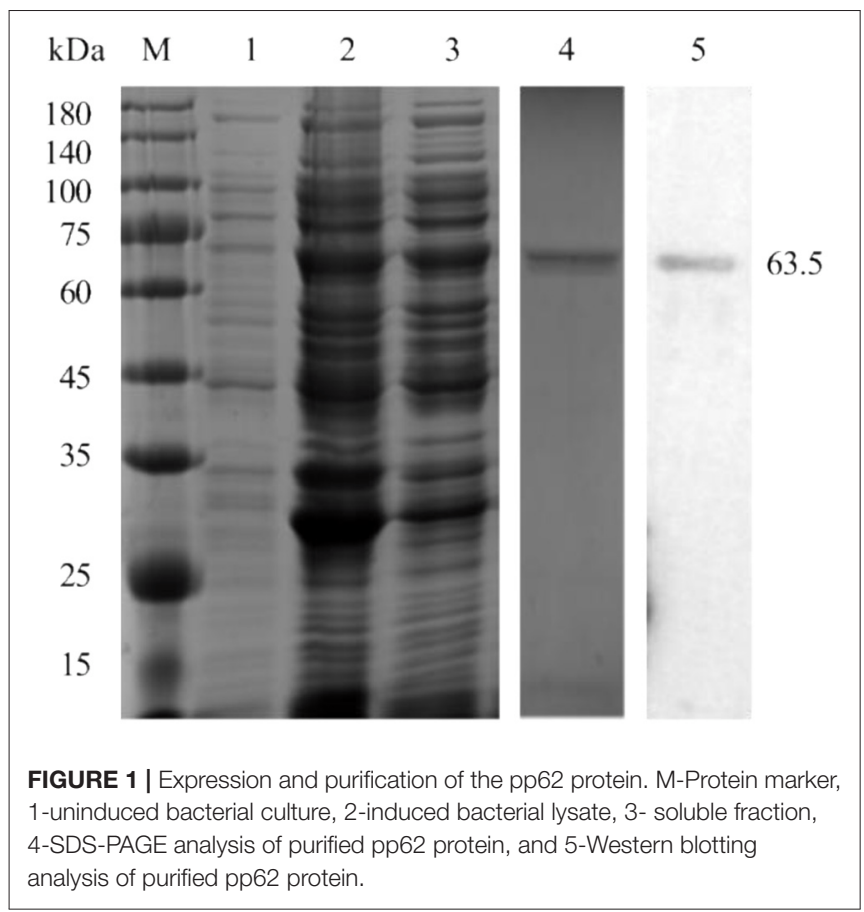

\section{Comparison of pp62-IELISA With Commercial Kits}

A total of 350 clinical serum samples were tested using pp62iELISA. Results were compared with commercial kits (ID. Vet, France) to evaluate the performance of pp62-iELISA in terms of relative sensitivity [(true positive/(true positive + false negative)]* $100 \%$ and relative specificity [(true negative/(true negative + false positive) $]^{*} 100 \%$.

\section{Statistical Analysis}

All data were analyzed using the Prism 5 software (GradphPad Software, La Jolla, CA, USA). All data were analyzed using a two-tailed student's $t$-test. $P<0.05$ was considered statistically significant.

\section{RESULTS}

\section{Expression and Purification of pp62 Protein} pp62 protein $(63.5 \mathrm{kDa})$ was successfully expressed in the soluble fraction and confirmed by Western blot analysis using ASFVpositive pig serum (Figure 1).

\section{Optimization of the Working Conditions of pp62-IELISA}

Checkerboard titration was applied to investigate the concentration of the coating antigen and sera. The maximum $P / N$ (6.08) value was obtained when the concentration of pp62 protein was $2 \mu \mathrm{g} / \mathrm{mL}$ and the dilution of serum was 1:400 (Table 1). Furthermore, other reaction conditions of the developed ELISA were optimized. In brief, the optimum coating condition was $2 \mathrm{~h}$ at $37^{\circ} \mathrm{C}$. The best blocking solution was selected as 5\% skimmed milk in PBST. The optimal reaction
TABLE 1 | Results of the $P / N$ values at different conditions.

\begin{tabular}{cccccc}
\hline \multirow{2}{*}{$\begin{array}{c}\text { Antigen at different } \\
\text { concentration }(\mu \mathbf{g} / \mathbf{m L})\end{array}$} & \multicolumn{4}{c}{ Dilution of sera } \\
\cline { 2 - 6 } & & $\mathbf{1 : 5 0}$ & $\mathbf{1 : 1 0 0}$ & $\mathbf{1 : 2 0 0}$ & $\mathbf{1 : 4 0 0}$ \\
\hline \multirow{4}{*}{4} & $\mathrm{P}$ & 2.415 & 2.075 & 1.669 & 1.276 \\
& $\mathrm{~N}$ & 0.443 & 0.368 & 0.303 & 0.231 \\
& $\mathrm{P} / \mathrm{N}$ & 5.45 & 5.63 & 5.51 & 5.52 \\
& $\mathrm{P}$ & 2.261 & 1.827 & 1.396 & 1.083 \\
& $\mathrm{~N}$ & 0.396 & 0.337 & 0.241 & 0.178 \\
& $\mathrm{~N}$ & 5.71 & 5.42 & 5.79 & 6.08 \\
& $\mathrm{P} / \mathrm{N}$ & 1.934 & 1.537 & 1.074 & 0.773 \\
& $\mathrm{P}$ & 0.370 & 0.321 & 0.232 & 0.174 \\
& $\mathrm{~N}$ & 5.23 & 4.78 & 4.62 & 4.44 \\
0.5 & $\mathrm{P} / \mathrm{N}$ & 1.786 & 1.473 & 0.926 & 0.686 \\
& $\mathrm{P}$ & 0.329 & 0.288 & 0.225 & 0.165 \\
& $\mathrm{~N}$ & 5.43 & 5.11 & 4.12 & 4.16 \\
\hline & $\mathrm{P} / \mathrm{N}$ & & & &
\end{tabular}

TABLE 2 | Results of the repeatability assay for pp62-iELISA.

\begin{tabular}{lccccc}
\hline \multirow{2}{*}{ Sample number } & \multicolumn{2}{c}{ Inter-assay CV (\%) } & & \multicolumn{2}{c}{ Intra-assay CV (\%) } \\
\cline { 2 - 3 } \cline { 6 - 6 } & $\mathbf{X} \pm$ SD & CV (\%) & & $\mathbf{X} \pm$ SD & CV (\%) \\
\hline 1 & $1.045 \pm 0.010$ & 0.96 & & $0.999 \pm 0.038$ & 3.80 \\
2 & $0.415 \pm 0.019$ & 4.58 & & $0.440 \pm 0.015$ & 3.40 \\
3 & $1.437 \pm 0.023$ & 1.60 & & $1.452 \pm 0.017$ & 1.17 \\
4 & $0.201 \pm 0.003$ & 1.49 & & $0.236 \pm 0.005$ & 2.13 \\
5 & $1.186 \pm 0.024$ & 2.02 & & $1.214 \pm 0.043$ & 3.54 \\
\hline
\end{tabular}

times for serum, secondary antibodies, and TMB solution were 45,30 , and $10 \mathrm{~min}$, respectively. Finally, the best working dilution of the HRP anti-pig IgG was 1:7,500.

\section{Repeatability of the pp62-IELISA}

The intra-batch variation and inter-batch variation were used to validate the repeatability of the pp62-iELISA. The coefficients of variation $(\mathrm{CV})$ of both the intra-assay and inter-assay were lower than 10\% (Table 2). These results indicated that the pp62-iELISA has high repeatability and low variability.

\section{Determination of Cut-Off Value}

The mean $(\bar{X})$ OD450 value of negative sera $(N=40)$ was 0.283 , and the SD was 0.045 , resulting in a cut-off value of $0.418(\bar{X}$ $+3 \mathrm{SD}=0.418)$ (Figure 2A). Therefore, samples with OD450 values $\geq 0.418$ were considered as positive, and samples with OD450 values $<0.418$ were considered negative.

\section{Diagnostic Sensitivity and Specificity of pp62-IELISA}

The optimized indirect ELISA antibody detection method was used to test PRRSV, PCV2, CSFV, PRV, and FMDV antibodypositive pig serum, and the results showed that all pathogenpositive sera tested negative except for the ASFV-positive control (Figure 2B). The results show that the sensitivity of the ELISA 


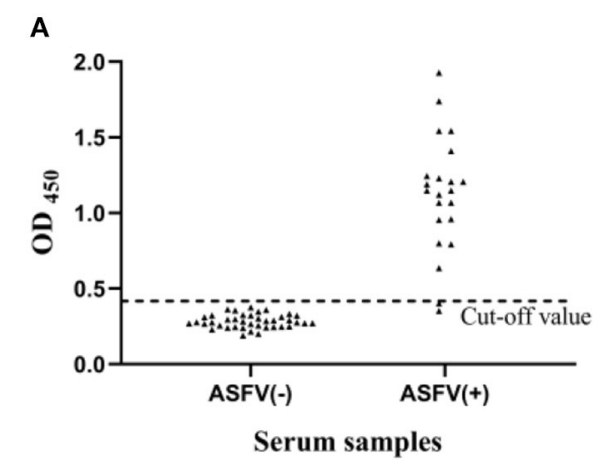

FIGURE 2 | Sensitivity and specificity of the pp62-iELISA. (A) Determination of the cut-off value of pp62-iELISA (OD $450=0.418)$. Distributions of OD values determined for ASFV-negative $(N=40)$ and ASFV-positive $(N=22)$ serum samples using pp62-iELISA; (B) the specificity test of pp62-iELISA. The pp62-iELISA detected no cross reactions with sera containing antibodies against five porcine pathogens; (C) sensitivity of the pp62-iELISA. ${ }^{\star \star \star} p<0.001$.
TABLE 3 | Results of pp62-iELISA in comparison with commercial kits.

\begin{tabular}{lcccc}
\hline & & \multicolumn{2}{c}{ ID. Vet } & Total \\
\cline { 3 - 4 } & & + & - & \\
\hline pp62-iELISA & + & 20 & 4 & 24 \\
& - & 2 & 324 & 326 \\
\hline Total & & 22 & 328 & 350 \\
\hline
\end{tabular}

Relative sensitivity $=20 / 22=90.91 \%$.

Relative specificity $=324 / 328=98.78 \%$.

Compliance rate $=344 / 350=98.29 \%$.

method established in this study is 1:1600 (Figure 2C), indicating that the sensitivity of the method is excellent.

\section{Evaluation of pp62-IELISA in Comparison With Commercial Kits}

The performance of pp62-iELISA in terms of relative sensitive, specificity, and accuracy were compared with commercial kits (ID. Vet, France). The results are shown in Table 3. The evaluation shows the good relative sensitivity and specificity of the tests, the pp62-iELISA had 90.90 and $98.78 \%$ sensitivity and specificity, respectively. The compliance rate was $98.28 \%$.

\section{DISCUSSION}

ASF is an acute, febrile, and highly lethal contagious viral disease of swine caused by ASFV (17). The disease has a short duration, high morbidity and mortality, and the highly virulent strain can cause mortality of up to $100 \%$ in domestic pigs and wild boars (18). The natural hosts of this virus include wild suids and arthropod vectors of the Ornithodoros genus. It is also the most serious infectious disease in the pig industry, causing significant economic losses to the swine raising industry and the pork market worldwide (19).

ASFV has a great number of genotypes, and its immune escape mechanism is complex and diverse, which leads to numerous difficulties in vaccine research (18). As there is no commercially available vaccine, molecular diagnostic methods and serological detection techniques are still considered as the main means of identifying infected animals and controlling ASF (20). What is more, the market urgently needs sensitive and accurate detection technology. Among them, ELISA serological diagnosis is not only a mature technology and stable detection method, but also has simple operation and low cost, so it is widely used in practice (21). There are three main ELISA kits from Ingenasa, Svanova, and ID. Vet, among which Ingenasa's blocking ELISA is the recommended serological test in Europe. However, Gallardo et al. compared the performance of ID. Vet's competition ELISA, Ingenasa's competition ELISA, and Svanova ELISA kits and indicated that the ID. Vet ELISA kit had a relatively low false positive rate and better performance (22). And in practical application, these three kits are expensive and stable supply cannot be guaranteed. With the emergence of mutated ASFV strains with weak virulence in various countries, nucleic acid fluorescence PCR combined with serum antibody detection is a common method to confirm diagnosis (23). Therefore, there is an urgent need to develop accurate serological antibody detection methods. Currently, serological diagnostic target proteins mainly focus on $\mathrm{p} 72, \mathrm{CD} 2 \mathrm{v}, \mathrm{p} 54, \mathrm{p} 30$, and pp62, which have strong antigenicity and high corresponding antibody titers in infected pigs (24). These proteins can serve as antigens to establish corresponding serological detection methods for antibody or antigen detection of ASFV.

pp62 protein is encoded by the ASFV CP530R gene with a molecular weight of $63.5 \mathrm{kDa}(25)$. As a polymeric protein, pp62 protein is co-assembled with another polymeric protein, pp220, to form the inner core shell of ASFV (15). The mature products of pp62, p35, and p15 participate in the icosahedron formation of the virus and are the basic components of the core and shell. Gallardo et al. used the arbovirus expression system to express ASFV pp62 protein, and thus established an indirect ELISA (iELISA) method (26). Further research indicated that pp62iELISA had better sensitivity and specificity than p30-iELISA and p54-iELISA, and could detect poorly preserved serum. Compared 
with the arbovirus expression system, the production cost of the prokaryotic expression system is relatively low, and the prepared protein is stable and easy to purify, which is suitable for largescale production $(27,28)$. Therefore, ASFV pp62 protein was expressed by the prokaryotic expression system in this study. The results showed that the recombinant protein was soluble and had strong reactivity. The recombinant protein was used as an ELISA coating antigen to establish and optimize the indirect ELISA detection method.

The specificity study shows that the developed assay does not cross react with antibodies of other related swine viruses such as PRRSV, PCV2, CSFV, PRV, and FMDV. Sensitivity studies showed that the method could detect commercial positive serum of ASFV at a maximum dilution of 1:1,600. The results of the repeatability test showed that the $\mathrm{CV}$ in both intra-batch and inter-batch repeated tests was $<10 \%$, indicating that the pp62iELISA established in this study had good repeatability. The coincidence rate with the ID. Vet ELISA kits reached 98.28\%. It can be applied to the detection of ASF antibody in clinical serum samples. This will play an important foundation for the further development of ASF antibody detection kits, and is of great significance to the prevention, control, and eradication of ASF.

\section{CONCLUSION}

The pp62-iELISA established in the present study was repeatable and specific for ASFV antibody detection, simple and economical

\section{REFERENCES}

1. Sang H, Miller G, Lokhandwala S, Sangewar N, Waghela SD, Bishop RP, et al. Progress toward development of effective and safe african swine fever virus vaccines. Front Vet Sci. (2020) 7:84. doi: 10.3389/fvets.2020.00084

2. Wu K, Liu J, Wang L, Fan S, Li Z, Li Y, et al. Current state of global african swine fever vaccine development under the prevalence and transmission of ASF in China. Vaccines. (2020) 8:30531. doi: 10.3390/vaccines8030531

3. Gaudreault NN, Madden DW, Wilson WC, Trujillo JD, Richt JA. African swine fever virus: an emerging DNA arbovirus. Front Vet Sci. (2020) 7:215. doi: 10.3389/fvets.2020.00215

4. Gavier-Widén D, Ståhl K, Dixon L. No hasty solutions for African swine fever. Science. (2020) 367:622-4. doi: 10.1126/science.aaz8590

5. Ge S, Li J, Fan X, Liu F, Li L, Wang Q, et al. Molecular characterization of African swine fever virus, China, 2018. Emerg Infect Dis. (2018) 24:2131-3. doi: $10.3201 /$ eid2411.181274

6. Teklue T, Sun Y, Abid M, Luo Y, Qiu HJ. Current status and evolving approaches to African swine fever vaccine development. Transbound Emerg Dis. (2020) 67:529-42. doi: 10.1111/tbed.13364

7. Sánchez-Vizcaíno JM, Mur L, Gomez-Villamandos JC, Carrasco L. An update on the epidemiology and pathology of African swine fever. J Comp Pathol. (2015) 152:9-21. doi: 10.1016/j.jcpa.2014.09.003

8. Ward MP, Tian K, Nowotny N. African Swine fever, the forgotten pandemic. Transbound Emerg Dis. (2021) 68:2637-9. doi: 10.1111/tbed.14245

9. Chapman DA, Darby AC, Da Silva M, Upton C, Radford AD, Dixon LK. Genomic analysis of highly virulent Georgia 2007/1 isolate of African swine fever virus. Emerg Infect Dis. (2011) 17:599-605. doi: 10.3201/eid1704.101283

10. Muñoz-Moreno R, Galindo I, Cuesta-Geijo M, Barrado-Gil L, Alonso C. Host cell targets for African swine fever virus. Virus Res. (2015) 209:118-27. doi: 10.1016/j.virusres.2015.05.026

11. Galindo I, Cuesta-Geijo MA, Hlavova K, Muñoz-Moreno R, Barrado-Gil L, Dominguez J, et al. African swine fever virus infects macrophages, the natural to produce and perform, and time-saving. The present report may facilitate the development of a reliable tool for the large-scale detection of ASFV antibodies.

\section{DATA AVAILABILITY STATEMENT}

Publicly available datasets were analyzed in this study. This data can be found at: https://www.ncbi.nlm.nih.gov/nuccore/ FR682468.2.

\section{AUTHOR CONTRIBUTIONS}

$\mathrm{XY}$ designed the study. $\mathrm{KZ}$ designed and prepared the tables and figures. ZD and DL made substantial, direct, and intellectual contributions to the work. $\mathrm{KZ}$ and $\mathrm{MZ}$ contributed to the writing of the paper. KZ, QY, and SF performed and collected data from the experiment and analyzed data. All authors approved the article for publication.

\section{FUNDING}

This study was supported by the Key Project of Research and Development Plan of Hunan Province (2019NK2171), the Natural Science Foundation of Hunan Province (2020JJ4041 and 2021JJ30316), the Outstanding Youth Scientist Foundation of Hunan Province (19B253), and the Youth Fund Project of Hunan Science and Technology Department (2020JJ5248). host cells, via clathrin- and cholesterol-dependent endocytosis. Virus Res. (2015) 200:45-55. doi: 10.1016/j.virusres.2015.01.022

12. Salas ML, Andrés G. African swine fever virus morphogenesis. Virus Res. (2013) 173:29-41. doi: 10.1016/j.virusres.2012.09.016

13. Dixon LK, Chapman DA, Netherton CL, Upton C. African swine fever virus replication and genomics. Virus Res. (2013) 173:3-14. doi: 10.1016/j.virusres.2012.10.020

14. Simón-Mateo C, Andrés G, Almazán F, Viñuela E. Proteolytic processing in African swine fever virus: evidence for a new structural polyprotein, pp62. J Virol. (1997) 71:5799-804. doi: 10.1128/jvi.71.8.5799-5804.1997

15. Andrés G, Alejo A, Salas J, Salas ML. African swine fever virus polyproteins pp220 and pp62 assemble into the core shell. J Virol. (2002) 76:12473-82. doi: 10.1128/JVI.76.24.12473-12482.2002

16. Alonso C, Miskin J, Hernáez B, Fernandez-Zapatero P, Soto L, Cantó C, et al. African swine fever virus protein p54 interacts with the microtubular motor complex through direct binding to light-chain dynein. J Virol. (2001) 75:9819-27. doi: 10.1128/JVI.75.20.9819-9827.2001

17. Wang Y, Xu L, Noll L, Stoy C, Porter E, Fu J, et al. Development of a real-time PCR assay for detection of African swine fever virus with an endogenous internal control. Transbound Emerg Dis. (2020) 67:2446-54. doi: $10.1111 /$ tbed. 13582

18. Wang T, Sun Y, Huang S, Qiu HJ. Multifaceted immune responses to African swine fever virus: implications for vaccine development. Vet Microbiol. (2020) 249:108832. doi: $10.1016 /$ j.vetmic.2020.108832

19. Liu Q, Ma B, Qian N, Zhang F, Tan X, Lei J, et al. Structure of the African swine fever virus major capsid protein p72. Cell Res. (2019) 29:953-5. doi: 10.1038/s41422-019-0232-x

20. Arias M, Jurado C, Gallardo C, Fernández-Pinero J, Sánchez-Vizcaíno JM. Gaps in African swine fever: analysis and priorities. Transbound Emerg Dis. (2018) 65(Suppl.1):235-47. doi: 10.1111/tbed.12695

21. Galindo I, Alonso C. African Swine fever virus: a review. Viruses. (2017) 9:50103. doi: 10.3390/v9050103 
22. Gallardo C, Nieto R, Soler A, Pelayo V, Fernández-Pinero J, MarkowskaDaniel I, et al. Assessment of African swine fever diagnostic techniques as a response to the epidemic outbreaks in Eastern European Union Countries: how to improve surveillance and control programs. J Clin Microbiol. (2015) 53:2555-65. doi: 10.1128/JCM.00857-15

23. Wang A, Jia R, Liu Y, Zhou J, Qi Y, Chen Y, et al. Development of a novel quantitative real-time PCR assay with lyophilized powder reagent to detect African swine fever virus in blood samples of domestic pigs in China. Transbound Emerg Dis. (2020) 67:284-97. doi: 10.1111/tbed.13350

24. Jia N, Ou Y, Pejsak Z, Zhang Y, Zhang J. Roles of African swine fever virus structural proteins in viral infection. J Vet Res. (2017) 61:135-43. doi: 10.1515/jvetres-2017-0017

25. Forth JH, Forth LF, Blome S, Höper D, Beer M. African swine fever wholegenome sequencing-Quantity wanted but quality needed. PLoS Pathog. (2020) 16:e1008779. doi: 10.1371/journal.ppat.1008779

26. Gallardo C, Blanco E, Rodríguez JM, Carrascosa AL, Sanchez-Vizcaino JM. Antigenic properties and diagnostic potential of African swine fever virus protein pp62 expressed in insect cells. J Clin Microbiol. (2006) 44:950-6. doi: 10.1128/JCM.44.3.950-956.2006

27. Laage R, Langosch D. Strategies for prokaryotic expression of eukaryotic membrane proteins. Traffic. (2001) 2:99-104. doi: 10.1034/ j.1600-0854.2001.020204.x
28. Sheibani N. Prokaryotic gene fusion expression systems and their use in structural and functional studies of proteins. Prep Biochem Biotechnol. (1999) 29:77-90. doi: 10.1080/10826069908544695

Conflict of Interest: The authors declare that the research was conducted in the absence of any commercial or financial relationships that could be construed as a potential conflict of interest.

Publisher's Note: All claims expressed in this article are solely those of the authors and do not necessarily represent those of their affiliated organizations, or those of the publisher, the editors and the reviewers. Any product that may be evaluated in this article, or claim that may be made by its manufacturer, is not guaranteed or endorsed by the publisher.

Copyright (c) 2022 Zhong, Zhu, Yuan, Deng, Feng, Liu and Yuan. This is an openaccess article distributed under the terms of the Creative Commons Attribution License (CC BY). The use, distribution or reproduction in other forums is permitted, provided the original author(s) and the copyright owner(s) are credited and that the original publication in this journal is cited, in accordance with accepted academic practice. No use, distribution or reproduction is permitted which does not comply with these terms. 